\title{
COMPLETENESS OF EIGENVECTORS IN BANACH SPACES
}

\author{
HAROLD E. BENZINGER
}

\begin{abstract}
We prove a general theorem on the completeness of the eigenvectors of linear operators in a Banach space. We then derive asymptotic estimates for the Green's functions of two-point boundary value problems which allow us to apply the above theorem to a wide class of such problems in the spaces $L^{p}(0,1), 1 \leqq p<\infty$.
\end{abstract}

1. Introduction. Let $B$ denote a Banach space, and let $B^{*}$ denote its dual. A sequence $\left\{\varphi_{k}\right\}$ of elements of $B$ is complete in $B$ if the collection of all finite sums $\sum \alpha_{k} \varphi_{k}, \alpha_{k}$ a scalar, is dense in $B$. The sequence $\left\{\varphi_{k}\right\}$ is closed in $B$ if the only element $\psi$ of $B^{*}$ for which $\psi\left(\varphi_{k}\right)=0$, all $k$, is the zero of $B^{*}$. It is easily seen that $\left\{\varphi_{k}\right\}$ is closed if and only if $\left\{\varphi_{k}\right\}$ is complete.

For the case that the scalar field is the complex field, we consider the problem of determining if a sequence $\left\{\varphi_{k}\right\}$ is complete in $B$, where the $\varphi_{k}$ 's arise as the eigenvectors and generalized eigenvectors of a linear operator $T: B \rightarrow B$. In the case that $B$ is a Hilbert space, there are completeness results provided that the resolvent operator is a Hilbert-Schmidt operator or an operator of class $C_{p}$, and the norm of the resolvent operator obeys certain growth conditions [1, pp. 1042, 1089, 1115]. These results are extended to Banach spaces in [9], [10].

If $T: B \rightarrow B$ has a compact resolvent $R(\lambda, T)$ for some $\lambda$, then the spectrum of $T$ is at most countably infinite, consisting entirely of eigenvalues $\lambda_{i}$ which are poles of $R(\lambda, T)$ [8, p. 416]. The invariant subspace corresponding to an eigenvalue $\lambda_{i}$ is of finite dimension $\nu_{i}$. By the operational calculus [7, pp. 287, 305], the projection $P_{i}$ of $B$ onto the invariant subspace corresponding to $\lambda_{i}$ has the form

$$
P_{i} f=\sum_{j=1}^{v_{i}} \psi_{i j}(f) \varphi_{i j}, \quad f \in B,
$$

where $\varphi_{i j} \in B, \psi_{i j} \in B^{*}$, and

$$
\psi_{i j}\left(\varphi_{k l}\right)=\delta_{i k} \delta_{j l} .
$$

In $\S 2$, we shall prove the following result.

Received by the editors January 11, 1972 and, in revised form, July 3, 1972. AMS (MOS) subject classifications (1970). Primary 47A70; Secondary 34B25. 
THEOREM 1.1. Let $T: B \rightarrow B$ be a densely defined linear operator with compact resolvent. Then the sequence $\left\{\varphi_{k l}\right\}$ is complete in $B$ provided that for each $r>0$ sufficiently large, the annulus $2 r \leqq|\lambda| \leqq 3 r$ contains a circle $C$ centered at the origin, lying entirely in the resolvent set of $T$, such that

$$
\|R(\lambda, T)\| \leqq K|\lambda|^{\mu}
$$

for $\lambda$ on $C$, where $K$ is a constant, and $\mu$ is an integer.

2. The completeness theorem. Since $T$ is densely defined in $B$, its adjoint $T^{*}: B^{*} \rightarrow B^{*}$ is well defined, and is a closed linear operator $[6, \mathrm{p} .43]$. Since $T$ and $T^{*}$ have the same resolvent sets we have $R\left(\lambda, T^{*}\right)=R^{*}(\lambda, T)$ [6, p. 56]. Thus the residue at $\lambda_{i}$ of $R\left(\lambda, T^{*}\right)$ is the adjoint $P_{i}^{*}$ of the residue of $R(\lambda, T)$ at $\lambda_{i}$, and $P_{i}^{*}$ has the form

$$
P_{i}^{*} g=\sum_{j=1}^{v_{i}} \varphi_{i j}(g) \psi_{i j}
$$

for $g$ in $B^{*}$. For convenience, we relabel the sequence $\left\{\varphi_{i j}\right\}$ as $\left\{\varphi_{k}\right\}$, and similarly for $\left\{\psi_{k}\right\}$. If $\left\{\varphi_{k}\right\}$ is not closed in $B$, there exists a nonzero $g$ in $B^{*}$ such that $\varphi_{k}(g)=0$ for all $k$. For such a $g, P_{i}^{*} g=0$ for all $i$. Using the biorthogonality (1.2), we see that the converse is true. Consequently $\left\{\varphi_{k}\right\}$ is closed if and only if the only element $g$ of $B^{*}$ for which $R\left(\lambda, T^{*}\right) g$ is entire is the zero of $B^{*}$. See also Definition 3 in $[8$, p. 443$]$ and the resulting discussion.

LeMma 2.1. Let $T: B \rightarrow B$ be a linear operator, and let $f$ be in $B, f \neq 0$. Then the equation

$$
T u=\lambda u+
$$

has no solution $u(\lambda)$ which is a polynomial in $\lambda$ on an infinite set $S$.

Proof. If we asume that $u(\lambda)=\sum_{k=0}^{m} \lambda^{k} u_{k}, u_{m} \neq 0$, is a solution of (2.2) for each $\lambda$, in $S$, then we easily see that each $u_{k}$ is in the domain of $T$. Substituting this expression into (2.2), we must have $u_{m}=0$, a contradiction.

If $\lambda$ is in the resolvent set of $T$, then the unique solution to (2.2) is

$$
u(\lambda)=-R(\lambda, T) f
$$

Thus any entire solution to (2.2) is an analytic continuation of $-R(\lambda, T) f$ onto the spectrum of $T$.

Proof of Theorem.1.1. Assume $\left\{\varphi_{k}\right\}$ is not closed in $B$. Then there exists an element $g$ in $B^{*}, g \neq 0$, such that $v(\lambda)=R\left(\lambda, T^{*}\right) g$ is entire. Let $\lambda_{0}$ be a fixed complex number, with $\left|\lambda_{0}\right|$ sufficiently large so that the 
annulus $2\left|\lambda_{0}\right| \leqq|\lambda| \leqq 3\left|\lambda_{0}\right|$ contains a circle $C$ on which $\left\|R\left(\lambda, T^{*}\right)\right\| \leqq$ $K|\lambda|^{\mu}$. Since $v(\lambda)$ is entire,

$$
v\left(\lambda_{0}\right)=\left(\frac{1}{2} \pi i\right) \int_{C}\left[v(\lambda) /\left(\lambda-\lambda_{0}\right)\right] d \lambda .
$$

Since $\left|\lambda-\lambda_{0}\right| \geqq\left|\lambda_{0}\right|$, and $|\lambda| \leqq 3\left|\lambda_{0}\right|$, we have

$$
\left\|v\left(\lambda_{0}\right)\right\| \leqq\left(\frac{1}{2} \pi\right) \int_{C}\left[\|v(\lambda)\| /\left|\lambda_{0}\right|\right]|d \lambda| \leqq 3 K\left|3 \lambda_{0}\right|^{\mu}\|g\|=K^{\prime}\left|\lambda_{0}\right|^{\mu} .
$$

Thus $-v(\lambda)$ is a polynomial solution to $T^{*} v=\lambda v+g$. By Lemma 2.1, this is not possible for $g \neq 0$, so $\left\{\varphi_{k}\right\}$ is closed in $B$.

COROLlaRY. If $B$ is reflexive, then under the assumptions of Theorem 1.1 , the sequence $\left\{\psi_{k}\right\}$ is complete in $B^{*}$.

Proof. If $\left\{\psi_{k}\right\}$ is not closed in $B^{*}$, then there exists $f$ in $B^{* *}=B, f \neq 0$, such that $P_{i} f=0$ for all $i$. The remainder of the discussion is as in the previous proof.

3. Completeness for ordinary differential operators. Let $l$ denote the $n$th order ordinary linear differential expression defined by

$$
l(u)=u^{(n)}+a_{n-1}(x) u^{(n-1)}+\cdots+a_{0}(x) u, \quad 0 \leqq x \leqq 1,
$$

where the $a_{j}$ 's are bounded measurable functions, and in addition $a_{n-1}^{(n-1)}$ exists and is also a bounded measurable function. Let $M, N$ denote two matrices of complex constants with $n$ linearly independent columns between them. Let $\hat{u}(x)$ denote the column vector $\left(u(x), u^{(1)}(x), \cdots\right.$, $\left.u^{(n-1)}(x)\right)$. Let

$$
U u=M \hat{u}(0)+N \hat{u}(1) .
$$

For $1 \leqq p<\infty$, let $\Delta=\Delta_{p}$ denote the subspace of $L^{p}(0,1)$ consisting of all functions $u$ of class $C^{n-1}[0,1]$ such that $u^{(n-1)}$ is absolutely continuous, $u^{(n)}$ is of class $L^{p}(0,1)$, and such that $U u=0$. Let $T: L^{p} \rightarrow L^{p}$ be defined on $\Delta$ by $T u=l(u)$. Since $\Delta$ contains all functions of class $C^{n}[0,1]$, which vanish, along with their first $n-1$ derivatives, at the endpoints, we see that $T$ is densely defined.

If $\lambda$ is in the resolvent set of $T$, then the solution to $T u=\lambda u+f, f$ in $L^{p}(0,1)$, is

$$
u(x, \lambda)=\int_{0}^{1} G(x, t, \lambda) f(t) d t=-R(\lambda, T) f,
$$

where $G$ is the Green's function of $T$.

Since $a_{n-1}^{(n-1)}$ is in $L^{\infty}[0,1]$, we can perform a substitution $u(x)=q(x) v(x)$, 
where

$$
q(x)=\exp \left[-(1 / n) \int_{0}^{x} a_{n-1}(t) d t\right],
$$

and obtain a new differential expression and boundary conditions for $v$. The significant feature of the transformed problem is that the coefficient of $v^{(n-1)}$ is zero. This simplifies the discussion of the asymptotic nature of solutions to $l(u)=\lambda u$.

Definition 3.1. The differential operator $T$ is Stone regular if the transformed problem satisfies Definition 3.1 in [2, p. 487].

If $G^{\prime}(x, t, \lambda)$ denotes the Green's function of the transformed problem, then as observed in [3, equation 2.5],

$$
G(x, t, \lambda)=q(x) G^{\prime}(x, t, \lambda) q^{-1}(t),
$$

where in [3] we used the substitution $\lambda=-\rho^{n}$. Thus we shall dispense with the distinction between the original problem and its transformed version, and assume that $a_{n-1} \equiv 0$.

The location of the eigenvalues of $T$ is discussed in [2, p. 489]. It is convenient for this purpose to refer to the $\rho$-plane. We will use the notation of [2], in particular the sectors $S_{i}$ are defined on p. 483, and the constants $\sigma$ and $\tau$ are defined on p. 485. Let $\delta>0$ be given. It is clear from the discussion in [2] that if each $\rho \in S_{i}$ such that $-\rho^{n}$ is an eigenvalue of $T$ is centered at a disc of radius $\delta$, then for $r>0$ sufficiently large, each region in $S_{i}$ of the form $(2 r)^{1 / n} \leqq|\rho| \leqq(3 r)^{1 / n}$ contains many circular arcs centered at the origin of the $\rho$-plane, and not intersecting any of the discs. The image in the $\lambda$-plane of such an arc is a circle $C$, centered at the origin of the $\lambda$-plane, contained entirely in the resolvent set of $T$, and satisfying $2 r \leqq|\lambda| \leqq 3 r$.

THEOREM 3.1. If the differential operator $T$ is Stone regular, there exists an integer $m \geqq 0$ such that

$$
n \rho^{n-1} G(x, t, \rho)=\rho^{m} O(1)
$$

as $|\rho| \rightarrow \infty$ in $S_{1}^{\prime}$ where the $O(1)$ term is uniform in $t$ and $x$ for $0 \leqq t, x \leqq 1$.

Proof. This is a direct consequence of equations (2.9) and (4.7) in [2, pp. 484, 492].

COROLlaRY. If $\lambda=-\rho^{n}$ is in the resolvent set of $T$, and if $\left|\rho-\rho_{0}\right| \geqq \delta$ for each eigenvalue $\lambda_{0}=-\rho_{0}^{n}$, then for $|\lambda|$ sufficiently large,

$$
|G(x, t, \lambda)| \leqq K|\lambda|^{(m+1-n) / n}, \quad 0 \leqq t, x \leqq 1,
$$

where $K$ is a constant.

Proof. This is a direct consequence of equations (3.4) and (3.5). 
We note at this point that there is no theoretical limit to the size of $m$. See [2, Theorem 5.3]. Let $\mu$ denote the first integer no smaller than $(m+1-n) / n$.

THEOREM 3.2. If $T$ is Stone regular, then for each $\lambda=-\rho^{n}$ in the resolvent set of $T$ such that $\left|\rho-\rho_{0}\right| \geqq \delta$ for each eigenvalue $\lambda_{0}=-\rho_{0}^{n}$, as an operator from $L^{p}$ to $L^{p}$,

$$
\|R(\lambda, T)\| \leqq K|\lambda|^{\mu} .
$$

Proof. By (3.6), we see that $G$, as a function to $t$, is of class $L^{\infty}(0,1)$, for fixed $x$ and $\lambda$. Thus $G$ is in $L^{q}(0,1)$ for each $q, 1 \leqq q \leqq \infty$. If $f$ is in $L^{p}(0,1), 1 \leqq p<\infty$, and if $p+q=p q$, then by Hölder's inequality,

$$
|u(x, \lambda)| \leqq\left[\int_{0}^{1}|G(x, t, \lambda)|^{q} d t\right]^{1 / q}\|f\|_{p} \leqq K|\lambda|^{\mu}\|f\|_{p} .
$$

Thus $\|u(\cdot, \lambda)\|_{p} \leqq K|\lambda|^{\mu}\|f\|_{p}$.

REMARK. In particular, (3.7) holds on each circle $C$ which is the image of a circular arc lying entirely in $S_{i}^{\prime}$.

THEOREM 3.3. If $T$ is Stone regular, the eigenfunctions and generalized eigenfunctions of $T$ form a sequence which is complete in $L^{p}(0,1)$ for $1 \leqq p<\infty$.

COROLlary. The eigenfunctions and generalized eigenfunctions of $T^{*}$ are complete in $L^{p}(0,1)$ for $1<p<\infty$.

REMARK. The adjoint in $L^{q}$ of a two-point boundary value problem in $L^{p}$ is known to be another two-point boundary value problem $(1<p<\infty)$, provided that the coefficients $a_{j}$ are sufficiently differentiable [5], so in such a case the corollary provides no new information. If the $a_{j}$ 's are not sufficiently differentiable, the $L^{p}$ adjoint of $T$ is a quasi-differential operator [4, p. 888]. Thus in these cases the corollary provides new information.

\section{REFERENCES}

1. N. Dunford and J. T. Schwartz, Linear operators. II: Spectral theory. Selfadjoint operators in Hilbert space, Interscience, New York, 1963. MR 32 \#6181.

2. H. E. Benzinger, Green's function for ordinary differential operators, J. Differential Equations 7 (1970), 478-496.

3. - The $L^{p}$ behavior of eigenfunction expansions, Trans. Amer. Math. Soc. 174 (1972), 333-344.

4. I. Halperin, Closures and adjoints of linear differential operators, Ann. of Math. (2) 38 (1937), 880-919.

5. G. C. Rota, Extension theory of differential operators. I, Comm. Pure Appl. Math. 11 (1958), 23-65. MR 20 \#3334. 
6. E. Hille and R. S. Phillips, Functional analysis and semi-groups, Amer. Math. Soc. Colloq. Publ., vol. 31, Amer. Math. Soc., Providence, R.I., 1957. MR 19, 664.

7. A. E. Taylor, Introduction to functional analysis, Wiley, New York; Chapman \& Hall, London, 1958. MR 20 \#5411.

8. J. T. Schwartz, Perturbations of spectral operators, and applications. I. Bounded perturbations, Pacific J. Math. 4 (1954), 415-458. MR 16, 144.

9. A. S. Markus, Some criteria for the completeness of a system of root vectors of a linear operator and for the summability of series in this system, Dokl. Akad. Nauk SSSR 155 (1964), 753-756=Soviet Math. Dokl. 5 (1964), 505-509. MR 28 \#5345.

10. - Certain criteria for the completeness of a system of root-vectors of a linear operator in a Banach space, Mat. Sb. 70 (112) (1966), 526-561; English transl., Amer. Math. Soc. Transl. (2) 85 (1969), 51-92. MR 35 \#7151.

Department of Mathematics, University of Illinois, Urbana, Illinois 61801 